\title{
Frailty prevalence and correlated risk in older diabetic patients of a diabetes clinic
}

Yuan-Ching Liu ${ }^{\mathrm{a}, \mathrm{b}}$, Neng-Chun Yu ${ }^{\mathrm{b}}$ I-Ying Chiub ${ }^{\mathrm{b}}$, Ching-Yi Lin ${ }^{\mathrm{b}}$, Cheng-Wei Lin ${ }^{\mathrm{b}}$, Hsiao-Wei Lu ${ }^{b}$, Wan-Yu Wu ${ }^{b}$, Wan-Ting Huang ${ }^{b}$, Yi-Hui Li ${ }^{b}$, Jackson Pui Man Waic,

\author{
${ }^{a}$ Department of Nursing, College of Medicine, National Taiwan University, Taipei, Taiwan \\ ${ }^{b}$ Neng-Chun Diabetes Clinic, Yilan County, Taiwan \\ Graduate Institute of Sport Science, National Taiwan Sport University, Taoyuan, Taiwan
}

\section{Background:}

Frailty is characterized by multi-system decline and vulnerability to adverse outcomes caused by relatively minor stresses. Older patients with diabetic mellitus (DM) were likely to develop frailty leading to higher mortality risk. The goal of this study was to investigate prevalence of frailty and its association with clinical manifestations, functional ability, depression, and cognitive impairment among 65-year and older type 2 DM patients.

\section{Methods:}

A total of 248 patients with type 2 diabetes were selected by random systematic sampling from a diabetic clinic located at Yilan County, Taiwan between August, 2015 and April, 2016. Participants consisted of 50.0\% male with age $73.9 \pm 5.9$ years (mean $\pm \mathrm{SD}$ ), body mass index $25.4 \pm 3.8$ $\mathrm{kg} / \mathrm{m}^{2}$, DM duration $15.2 \pm 9.0$ years, glycosylated hemoglobin $7.1 \pm 1.1 \%$, type of medication use $3.1 \pm 1.5$, and number of self-reported clinical mani-

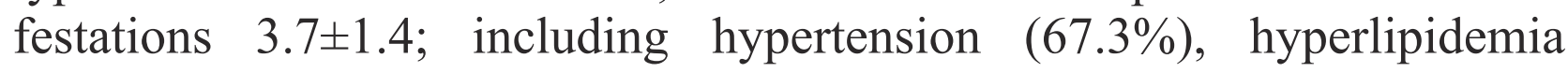
(75.4\%), diabetic nephropathy $(52.0 \%)$, retinopathy (22.6\%), neuropathy $(9.3 \%)$, and stroke $(3.6 \%)$. The self-reported frequency of fall was $0.6 \pm 1.2$, hypoglycemia $(<70 \mathrm{mg} / \mathrm{dl})$ was $2.3 \pm 5.7$, and hyperglycemia $(>300 \mathrm{mg} / \mathrm{dl})$ was 2.1 \pm 8.6 in the past year (Table 1). Fried et al.'s phenotype model - unintentional weight loss, self-reported exhaustion, low physical activity, slow walking speed, and muscle weakness (grip strength) was adopted to set our five criteria based on participants' physical characteristics (height, body mass, and body mass index) because of smaller-size Chinese and different physical activity habit, as such a cutoff of $\geq 3 \mathrm{~kg}$ or $\geq 5 \%$ was used for weight loss in the past year and the Chinese version International Physical Activity Questionnaire was used for assessment of activity energy expenditure in the past week. Frailty was defined by the presence of three or more of the criteria. Functional ability was evaluated by the Activities of Daily Living (ADL) scale and Instrumental ADL (IADL) scale. Depression was evaluated by the Taiwan Geriatric Depression Scale (TGDS) and cognitive impairment by Mini-Mental State Examination (MMSE) . Demographic and clinical data was obtained from medical records. The independent-samples t-test or nonparametric Manne Whitney U-test was used to examine difference in means and $\chi^{2}$ test in frequency between two variables. Multivariate logistic regression was used to identify the correlates of frailty.

\section{Results:}

The frailty prevalent rate was $9.3 \%$ among all participants, $9.6 \%$ among males, and $8.9 \%$ among females. The age-specific frailty prevalent rate was $5.8 \%, 12.2 \%, 23.1 \%$, for $65-74.9,75-84.9$, and $\geq 85$ years respectively (Table $2 \& 3$ ). Compared to patients without frailty, patients with frailty had significantly $(p<0.05)$ higher percentage of stroke $(13.0 \% \mathrm{vs}$. $2.7 \%)$, diabetic nephropathy $(78.3 \%$ vs. $49.3 \%)$ and fall $(1.1 \pm 1.2$ vs. $0.5 \pm 1.2$ ), lower physical function ( $\geq 1$ IADL task: $39.1 \%$ vs. $14.7 \%$ ), higher depression level (5.2 \pm 3.5 vs. 2.0 \pm 2.4 ), and greater cognitive impairment (22.1 \pm 4.0 vs. $25.5 \pm 4.3)$ (Table1). Independent correlates of frailty among participants were age $\geq 85$ years (adjusted odd ratio, OR, 6.42;95\% CI, 1.21-34.1; $\mathrm{p}=0.029$ ), diabetic nephropathy (adjusted OR, 3.98; 95\% CI, 1.10-14.30; $\mathrm{p}=0.035$ ), higher depression level (adjusted OR, 1.48; 95\% CI, $1.24-1.76 ; \mathrm{p}<0.001$ ), and longer diabetes duration (adjusted OR, 1.06; 95\% CI, 1.01-1.12; $\mathrm{p}=0.028$ ).

\section{Conclusions:}

About one-tenth of the diabetic patients studied, irrespective of gender, were frail. Frailty increased with advancing age - approximately one-fourth by $\geq 85$ years old. Besides old age and longer DM duration, having diabetic nephropathy and depression symptoms increased the frailty risk. Early detection by routine screening for sign of depression or chronic kidney disease is strongly recommended in diabetic care for the prevention or delay of frailty.

\section{Reference:}

Fried, L. P., Tangen, C. M., Walston, J., Newman, A. B., Hirsch, C., Gottdiener, J., Burke, G. (2001) Frailty in older adults: evidence for a phenotype. The Journals of Gerontology Series A Biological Sciences and Medical Sciences, 56(3), M146-M157.
Table 1. Characteristics of the study participants

$\mathrm{N}=248$

\begin{tabular}{|c|c|c|c|c|}
\hline Variables & $\begin{array}{c}\text { Total } \\
\mathbf{N}=\mathbf{2 4 8} \\
\mathbf{N}(\%) / \text { Mean } \pm \text { SD }\end{array}$ & $\begin{array}{c}\text { Non-frail } \\
\mathrm{N}=\mathbf{2 2 5}(\mathbf{9 0 . 7 \% )} \\
\mathrm{N}(\%) / \text { Mean } \pm \text { SD }\end{array}$ & $\begin{array}{c}\text { Frail } \\
\mathbf{N}=23(9.3 \%) \\
\mathbf{N}(\%) / \text { Mean } \pm \text { SD }\end{array}$ & p value \\
\hline Age & $73.9 \pm 5.9$ & $73.6 \pm 5.8$ & $77.0 \pm 6.1$ & 0.013 \\
\hline $65-74.9$ & $137(55.2 \%)$ & $129(57.3 \%)$ & $8(34.8 \%)$ & \\
\hline $75.0-84.9$ & $98(39.5 \%)$ & $86(38.2 \%)$ & $12(52.2 \%)$ & 0.053 \\
\hline$\geqq 85$ & $13(5.2 \%)$ & $10(4.4 \%)$ & $3(13.0 \%)$ & \\
\hline DM duration & $15.2 \pm 9.0$ & $14.7 \pm 8.6$ & $19.7 \pm 11.5$ & 0.056 \\
\hline \multicolumn{5}{|l|}{ Sex } \\
\hline Male & $124(50.0 \%)$ & $112(49.8 \%)$ & $12(52.2 \%)$ & 0.827 \\
\hline Female & $124(50.0 \%)$ & $113(50.2 \%)$ & $11(47.8 \%)$ & \\
\hline \multicolumn{5}{|l|}{ Education status } \\
\hline Illiterate & $60(24.2 \%)$ & $52(23.1 \%)$ & $8(34.8 \%)$ & \\
\hline Primary school & $109(44.0 \%)$ & $100(44.4 \%)$ & $9(39.1 \%)$ & \\
\hline Junior high school & $22(8.9 \%)$ & $19(8.4 \%)$ & $3(13.0 \%)$ & 0.227 \\
\hline Senior high school & $37(14.9 \%)$ & $36(16.0 \%)$ & $1(4.3 \%)$ & \\
\hline College & $17(6.9 \%)$ & $16(7.1 \%)$ & $1(4.3 \%)$ & \\
\hline Graduated school & $3(1.2 \%)$ & $2(0.9 \%)$ & $1(4.3 \%)$ & \\
\hline \multicolumn{5}{|l|}{ Marital status } \\
\hline Single & $1(0.4 \%)$ & $0(0 \%)$ & $1(4.3 \%)$ & \\
\hline Married & $180(72.6 \%)$ & $163(72.4 \%)$ & $17(73.9 \%)$ & 0.164 \\
\hline Divorce & $1(0.4 \%)$ & $1(0.4 \%)$ & $0(0.0 \%)$ & \\
\hline Widow & $66(26.6 \%)$ & $61(27.1 \%)$ & $5(21.7 \%)$ & \\
\hline \multicolumn{5}{|l|}{ Living status } \\
\hline Living alone & $20(8.1 \%)$ & $19(8.4 \%)$ & $1(4.3 \%)$ & 0.704 \\
\hline Living with family & $228(91.9 \%)$ & $206(91.6 \%)$ & $22(95.7 \%)$ & \\
\hline \multicolumn{5}{|l|}{ Clinical parameters } \\
\hline A1C & $7.1 \pm 1.1$ & $7.1 \pm 1.1$ & $7.2 \pm 0.8$ & 0.243 \\
\hline $\mathrm{A} 1 \mathrm{C}<8 \%$ & $211(85.1 \%)$ & $192(85.3 \%)$ & $19(82.6 \%)$ & \\
\hline $\mathrm{A} 1 \mathrm{C} \geqq 8 \%$ & $37(14.9 \%)$ & $33(14.7 \%)$ & $4(17.4 \%)$ & 0.758 \\
\hline Total cholesterol $(\mathrm{N}=246)$ & $156.7 \pm 29.0$ & $156.1 \pm 27.8(\mathrm{~N}=223)$ & $162.0 \pm 39.5(\mathrm{~N}=23)$ & 0.933 \\
\hline HDL $(\mathrm{N}=246)$ & $52.1 \pm 15.8$ & $52.3 \pm 16.2$ & $50.2 \pm 10.2$ & 0.775 \\
\hline LDL $(\mathrm{N}=247)$ & $77.9 \pm 22.9$ & $78.0 \pm 22.1$ & $77.4 \pm 29.6$ & 0.363 \\
\hline Triglycerides $(\mathrm{N}=246)$ & $139.0 \pm 89.5$ & $138.5 \pm 91.6$ & $144.4 \pm 67.4$ & 0.165 \\
\hline Creatinine $(\mathrm{N}=246)$ & $1.1 \pm 04.8$ & $1.1 \pm 0.8$ & $1.5 \pm 0.9$ & 0.007 \\
\hline ACR (N=233) & $104.3 \pm 277.5$ & $90.1 \pm 242.6$ & $263.9 \pm 516.8$ & 0.165 \\
\hline$<\mathbf{3 0}$ & $148(63.5 \%)$ & $137(64.0 \%)$ & $11(57.9 \%)$ & \\
\hline 30-300 & $64(27.5 \%)$ & $60(28.0 \%)$ & $4(21.1 \%)$ & 0.154 \\
\hline$>300$ & $21(9.0 \%)$ & $17(7.9 \%)$ & $4(21.1 \%)$ & \\
\hline eGFR $(N=246)$ & $69.4 \pm 20.5$ & $70.8 \pm 19.6$ & $56.8 \pm 24.9$ & 0.005 \\
\hline$<60$ & $80(32.5 \%)$ & $67(30.0 \%)$ & $13(56.5 \%)$ & 0010 \\
\hline$\geqq 60$ & $166(67.5 \%)$ & $156(70.0 \%)$ & $10(43.5 \%)$ & 0.010 \\
\hline BMI & $25.4 \pm 3.8$ & $25.2 \pm 3.6$ & $26.7 \pm 5.4$ & 0.268 \\
\hline \multicolumn{5}{|l|}{ Medical history } \\
\hline Hypertension & $167(67.3 \%)$ & $152(67.6 \%)$ & $15(65.2 \%)$ & 0.820 \\
\hline Cardiovascular diseases & $63(25.4 \%)$ & $57(25.3 \%)$ & $6(26.1 \%)$ & 0.937 \\
\hline Hyperlipidemia & $187(75.4 \%)$ & $167(74.2 \%)$ & $20(87.0 \%)$ & 0.177 \\
\hline Stroke & $9(3.6 \%)$ & $6(2.7 \%)$ & $3(13.0 \%)$ & 0.041 \\
\hline Diabetic nephropathy & $129(52.0 \%)$ & $111(49.3 \%)$ & $18(78.3 \%)$ & 0.008 \\
\hline Retinopathy & $56(22.6 \%)$ & $50(77.8 \%)$ & $6(73.9 \%)$ & 0.673 \\
\hline Neuropathy & $23(9.3 \%)$ & $23(10.2 \%)$ & $0(0 \%)$ & 0.143 \\
\hline Average disease number & $3.7 \pm 1.4$ & $2.8 \pm 1.4$ & $3.2 \pm 1.2$ & 0.080 \\
\hline Types of medicine & $3.1 \pm 1.6$ & $3.1 \pm 1.5$ & $3.7 \pm 1.7$ & 0.109 \\
\hline Frequency of fall & $0.6 \pm 1.2$ & $0.5 \pm 1.2$ & $1.1 \pm 1.2$ & 0.001 \\
\hline Frequency of hypoglycemia & $3.2 \pm 6.0$ & $3.1 \pm 5.8$ & $4.0 \pm 7.6$ & 0.499 \\
\hline $\begin{array}{l}\text { Frequency of hyperglycemia } \\
(>\mathbf{3 0 0} \mathbf{~ m g / d l})\end{array}$ & $2.1 \pm 8.6$ & $2.1 \pm 8.9$ & $2.2 \pm 4.7$ & 0.042 \\
\hline \multicolumn{5}{|l|}{ Functional assessment } \\
\hline ADL & $98.6 \pm 4.7$ & $98.9 \pm 2.8$ & $95.4 \pm 12.3$ & 0.198 \\
\hline$\geqq 1$ IADL task disability & $42(16.9 \%)$ & $33(14.7 \%)$ & $9(39.1 \%)$ & 0.003 \\
\hline TGDS & $2.3 \pm 2.6$ & $2.0 \pm 2.4$ & $5.2 \pm 3.5$ & $<0.001$ \\
\hline MMSE & $25.2 \pm 4.4$ & $25.5 \pm 4.3$ & $22.1 \pm 4.0$ & $<0.001$ \\
\hline
\end{tabular}

Table2. Prevalence of frailty

\begin{tabular}{lccc}
\hline Age group (n) & $\begin{array}{r}\text { Overall } \\
\mathbf{( N = 2 4 8 )} \\
\text { \% Frail }\end{array}$ & $\begin{array}{c}\text { Men } \\
(\mathbf{N}=\mathbf{2 5}) \\
\text { \% Frail }\end{array}$ & $\begin{array}{r}\text { Women } \\
(\mathbf{N}=123) \\
\text { \% Frail }\end{array}$ \\
\hline $65-74.9(\mathrm{n}=137)$ & $5.8 \%$ & $4.3 \%$ & $7.5 \%$ \\
$75-84.9(\mathrm{n}=98)$ & $12.2 \%$ & $12.2 \%$ & $12.2 \%$ \\
$\geqq 85 \quad(\mathrm{n}=13)$ & $23.1 \%$ & $50.0 \%$ & $0.0 \%$ \\
Total & $9.3 \%$ & $9.6 \%$ & $8.9 \%$ \\
\hline \% Frailty was defined by the presence of three or more of the criteria
\end{tabular}

※ Frailty was defined by the presence of three or more of the criteria.

Table3. Prevalence of frailty phenotype components in percentage

\begin{tabular}{lccc}
\hline & $\begin{array}{c}\text { Total } \\
(\mathbf{N = 2 4 8})\end{array}$ & $\begin{array}{c}\text { Men } \\
(\mathbf{N}=\mathbf{1 2 5})\end{array}$ & $\begin{array}{c}\text { Women } \\
(\mathbf{N = 1 2 3})\end{array}$ \\
\hline Frequency of frailty components & $\%$ & $\%$ & $\%$ \\
Grip strength (kg) & $19.8 \%$ & $19.2 \%$ & $20.3 \%$ \\
Slow walk (s) & $20.6 \%$ & $20.0 \%$ & $21.1 \%$ \\
Weight loss & $10.5 \%$ & $11.2 \%$ & $9.8 \%$ \\
Exhaustion & $19.8 \%$ & $17.6 \%$ & $22.0 \%$ \\
Low activity (kcal) & $20.2 \%$ & $20.0 \%$ & $20.3 \%$ \\
Number of frailty components present & & & \\
0 & $44.8 \%$ & $45.6 \%$ & $43.9 \%$ \\
1 & $30.6 \%$ & $30.4 \%$ & $30.9 \%$ \\
2 & $15.3 \%$ & $14.4 \%$ & $16 \% 3 \%$ \\
3 & $8.1 \%$ & $8.8 \%$ & $7.3 \%$ \\
4 & $0.8 \%$ & $0.8 \%$ & $0.8 \%$ \\
5 & $0.4 \%$ & $0.0 \%$ & $0.8 \%$ \\
\hline & & &
\end{tabular}

